\title{
Evidence of Air Dispersion: HFPO-DA and PFOA in Ohio and West Virginia Surface Water and Soil Near a Fluoropolymer Production Facility
}

\section{Supporting Information}

Jason E. Galloway, ${ }^{\dagger}$ Anjelica V. P. Moreno, ${ }^{\#}$ Andrew B. Lindstrom, ${ }^{\ddagger}$ Mark J. Strynar, ${ }^{\ddagger}$ Seth Newton,, Andrew A. May, $\#, \S$ and Linda K. Weavers $\#, \S^{*} *$

${ }^{\dagger}$ Department of Molecular Genetics, The Ohio State University, Columbus, Ohio 43210, United States

\# Environmental Science Graduate Program, The Ohio State University, Columbus, Ohio 43210, United States

*U.S. Environmental Protection Agency, Research Triangle Park, North Carolina 27711, United States

$\S$ Department of Civil, Environmental and Geodetic Engineering, The Ohio State University, Columbus, Ohio 43210, United States

* Corresponding Author: weavers.1@osu.edu 


\section{Table of Contents}

Materials

Figure S1. Sampling locations and PFAS concentrations in surface waters from the second sampling trip

Figure S2. Predicted wet+dry deposition rates from CALPUFF model output and measured soil concentrations from 2016 and 2018 sampling trips S4

Table S1. Method performance S5

Table S2. Duplicate comparison S5

Table S3. Measured PFAS surface waterconcentration results from the first sampling trip .S6

Table S4. Measured PFAS surface water concentration results from the second sampling trip S7

Table S5. Measured PFAS surface water concentration results from the third sampling trip S8

Table S6. PFASs concentrations in public water. S9

Table S7. Measured PFAS soil concentration results from soil sampling trips S9

Table S8. Sample metadata from the first sampling trip. S10

Table S9. Sample metadata from the second sampling trip S11

Table S10. Sample metadata from the third sampling trip. $\mathrm{S} 12$

Table S11. Sample metadata for soil samples $\mathrm{S} 13$ 


\section{Materials}

Targeted PFAS - perfluorobutanoic acid (PFBA), perfluoropentanoic acid (PFPeA), perfluorohexanoic acid (PFHxA), perfluoroheptanoic acid (PFHpA), perfluorooctanoic acid (PFOA), perfluorononanoic acid (PFNA), perfluorodecanoic acid (PFDA), perfluorobutane sulfonic acid (PFBS), perfluorohexane sulfonic acid (PFHxS), and perfluorooctane sulfonic acid (PFOS) - were purchased from Wellington Laboratories (Guelph, Ontario, Canada), and HFPO-DA was obtained from Synquest Laboratories (Alachua, FL). Isotopically labeled PFASs used for internal standards (IS) were obtained from Wellington Laboratories. Deionized (DI) water was generated in-house using a Barnstead EasyPure UV/UF lab water system (Dubuque, IA).
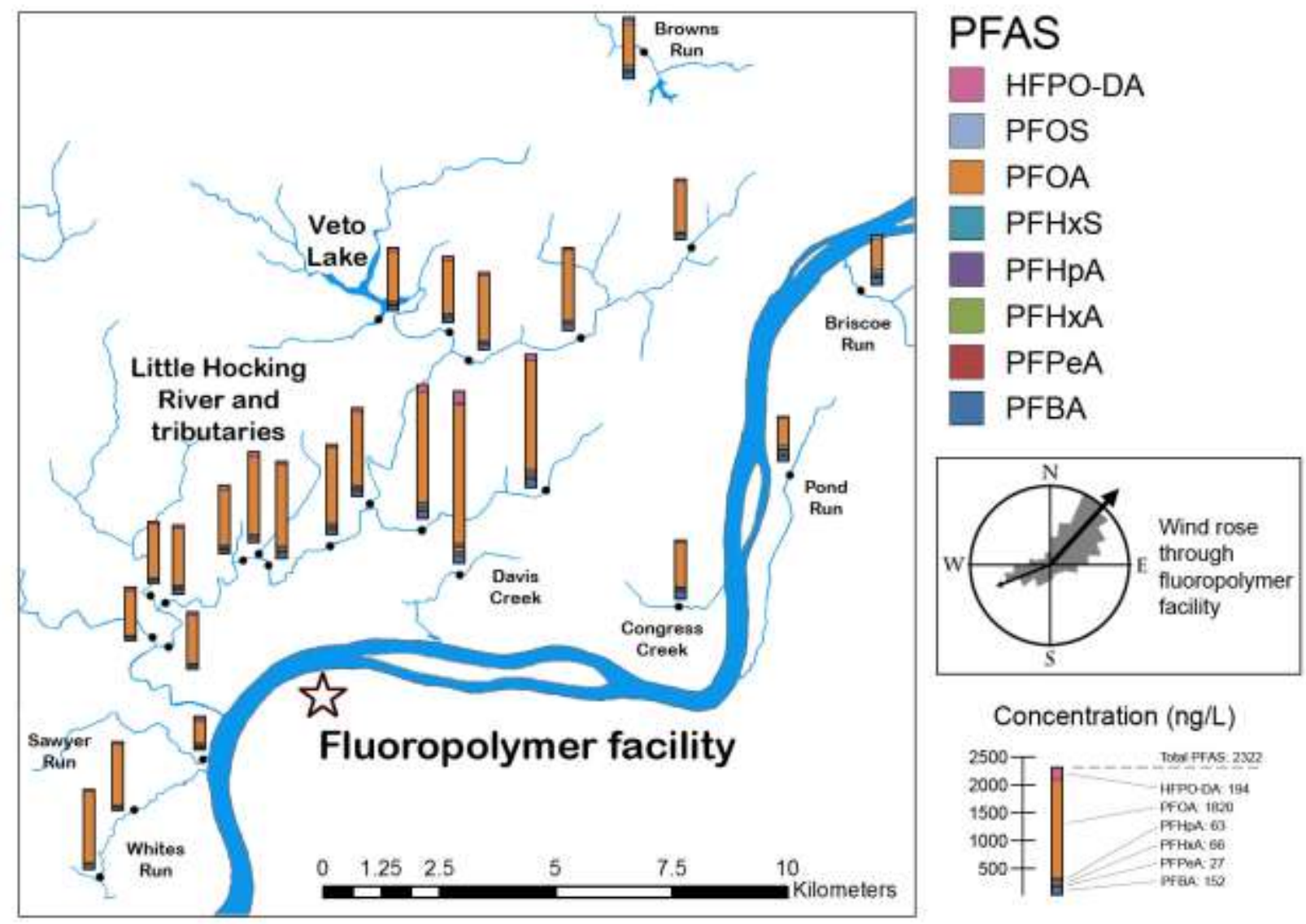

Figure S1. Sampling locations and PFAS concentrations in surface waters from the second sampling trip, July 12-

13, 2016. 


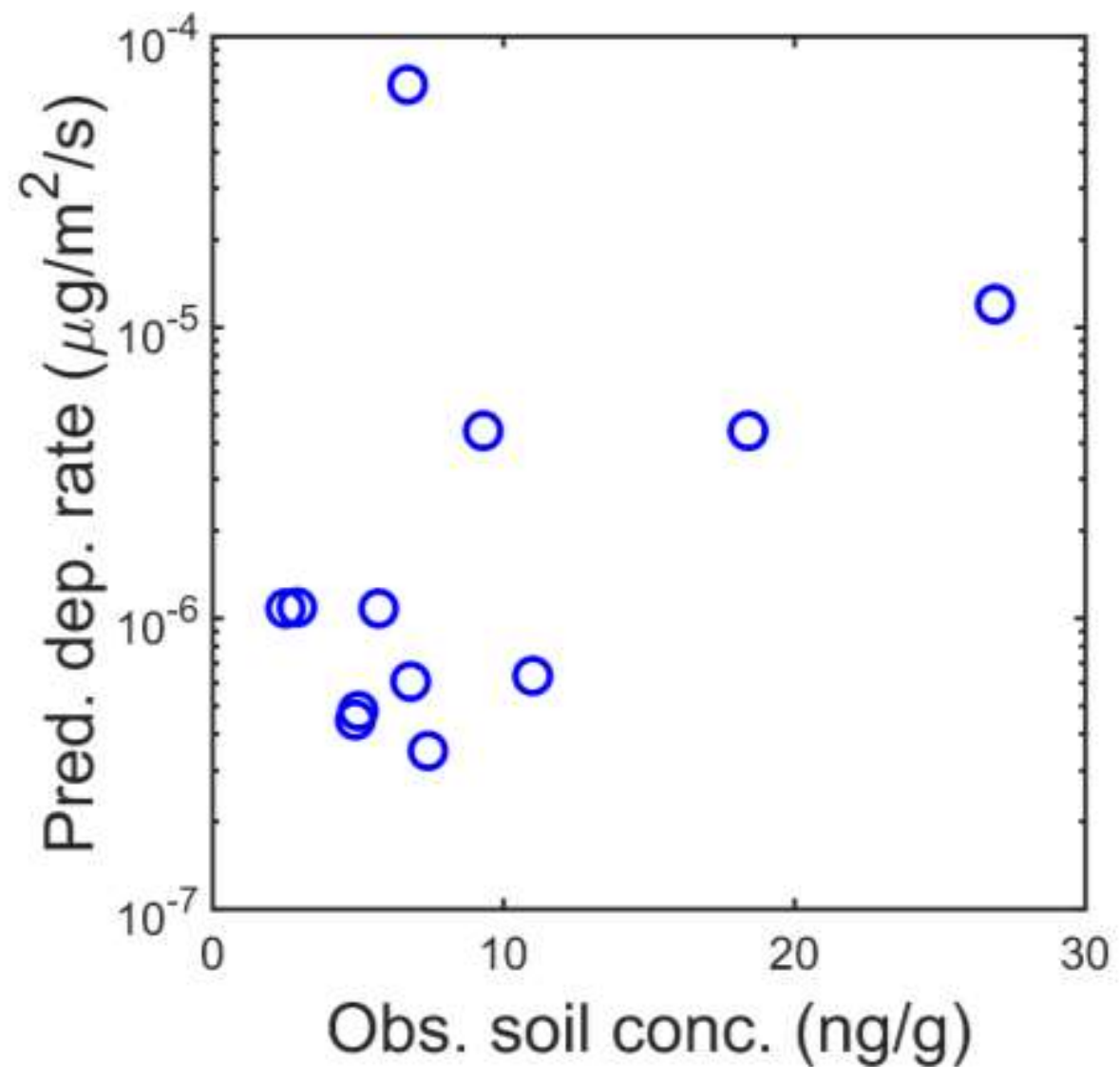

Figure S2. Predicted PFOA wet+dry deposition rates from CALPUFF model output and measured PFOA soil concentrations from 2016 and 2018 sampling trips. Predictions correspond to the exact locations of the soil samples. The value at $\left[7 \mathrm{ng} / \mathrm{g}, 6 \times 10^{-5} \mu \mathrm{g} / \mathrm{m}^{2} / \mathrm{s}\right]$ is a suspected outlier ( $p$-value of slope $=0.14$ with this point, or $<0.001$ without). 
Table S1. Method performance.

\begin{tabular}{|c|c|c|c|c|c|c|c|c|c|c|c|c|}
\hline & \multicolumn{4}{|c|}{ Sample Trip \#1 } & \multicolumn{4}{|c|}{ Sample Trip \#2 } & \multicolumn{4}{|c|}{ Sample Trip \#3 } \\
\hline & \multicolumn{2}{|c|}{$25 \mathrm{ng} / \mathrm{L}$} & \multicolumn{2}{|c|}{$100 \mathrm{ng} / \mathrm{L}$} & \multicolumn{2}{|c|}{$25 \mathrm{ng} / \mathrm{L}$} & \multicolumn{2}{|c|}{$100 \mathrm{ng} / \mathrm{L}$} & \multicolumn{2}{|c|}{$25 \mathrm{ng} / \mathrm{L}$} & \multicolumn{2}{|c|}{$100 \mathrm{ng} / \mathrm{L}$} \\
\hline & $\mathrm{ng} / \mathrm{L}$ & $\begin{array}{c}\% \\
\text { recovery }\end{array}$ & $\mathrm{ng} / \mathrm{L}$ & $\begin{array}{c}\% \\
\text { recovery }\end{array}$ & $\mathrm{ng} / \mathrm{L}$ & $\begin{array}{c}\% \\
\text { recovery }\end{array}$ & $\mathrm{ng} / \mathrm{L}$ & $\begin{array}{c}\% \\
\text { recovery }\end{array}$ & $\mathrm{ng} / \mathrm{L}$ & $\begin{array}{c}\% \\
\text { recovery }\end{array}$ & $\mathrm{ng} / \mathrm{L}$ & $\begin{array}{c}\% \\
\text { recovery }\end{array}$ \\
\hline PFBA & 27.4 & 110 & 92.0 & 92.0 & 13.3 & 53.3 & 96.2 & 96.2 & 34.8 & 139 & 101 & 101 \\
\hline PFPeA & 27.5 & 110 & 92.3 & 92.3 & 13.0 & 52.1 & 104 & 104 & 37.1 & 149 & 106 & 106 \\
\hline PFBS & 27.4 & 110 & 89.5 & 89.5 & - & - & - & - & 34.5 & 138 & 184 & 184 \\
\hline PFHxA & 25.3 & 101 & 79.3 & 79.3 & 23.1 & 92.2 & 99.3 & 99.3 & 31.2 & 125 & 84.6 & 84.6 \\
\hline PFHpA & 26.8 & 107 & 79.3 & 79.3 & 19.9 & 79.5 & 106 & 106 & 15.8 & 63.0 & 132 & 132 \\
\hline PFHXS & 24.9 & 99.8 & 89.7 & 89.7 & - & - & 47.2 & 47.2 & 29.5 & 118 & 148 & 148 \\
\hline PFOA & 24.4 & 97.5 & 91.3 & 91.3 & 17.4 & 69.7 & 94.7 & 94.7 & 16.8 & 67.1 & 129 & 129 \\
\hline PFNA & 25.6 & 102 & 87.3 & 87.3 & 20.1 & 80.3 & 89.8 & 89.8 & 10.6 & 42.4 & 55.1 & 55.1 \\
\hline PFOS & 25.5 & 102 & 88.3 & 88.3 & 20.4 & 81.6 & 97.7 & 97.7 & 25.5 & 102 & 109 & 109 \\
\hline PFDA & 23.4 & 93.6 & 79.0 & 79.0 & 17.7 & 70.7 & 108 & 108 & 27.2 & 109 & 221 & 221 \\
\hline $\begin{array}{l}\text { HFPO- } \\
\text { DA }\end{array}$ & - & - & - & - & - & - & - & - & 28.7 & 115 & 78.2 & 78.2 \\
\hline
\end{tabular}

Table S2. Duplicate comparison.

\begin{tabular}{|c|c|c|c|c|c|c|c|}
\hline \multirow[b]{2}{*}{ Description } & \multirow[b]{2}{*}{ Date } & \multicolumn{3}{|c|}{ PFOA } & \multicolumn{3}{|c|}{ HFPO-DA } \\
\hline & & $\begin{array}{c}\text { Sample } \\
\# 1(\text { ng/L) }\end{array}$ & $\begin{array}{c}\text { Sample } \\
\# 2(\mathrm{ng} / \mathrm{L})\end{array}$ & $\begin{array}{c}\% \\
\text { difference }\end{array}$ & $\begin{array}{c}\text { Sample } \\
\# 1(\text { ng/L) }\end{array}$ & $\begin{array}{c}\text { Sample } \\
\# 2(\mathrm{ng} / \mathrm{L})\end{array}$ & $\begin{array}{c}\% \\
\text { difference }\end{array}$ \\
\hline Lee Creek & $6 / 6 / 16$ & 50.9 & 65.3 & 24.8 & $*$ & $*$ & - \\
\hline $\begin{array}{l}\text { Sawyer Run (Ohio } \\
\text { River) }\end{array}$ & $6 / 8 / 16$ & $*$ & $*$ & - & - & $*$ & - \\
\hline $\begin{array}{l}\text { Vienna Island (Ohio } \\
\text { River) }\end{array}$ & $6 / 9 / 16$ & $*$ & $*$ & - & - & - & - \\
\hline Little Hocking \#4 & $7 / 12 / 16$ & 1770 & 1220 & 36.8 & 86 & 37 & 79.7 \\
\hline Veto Lake & $12 / 1 / 16$ & 412 & 321 & 24.8 & 31.0 & 37.5 & 19.0 \\
\hline Mill Branch & $12 / 2 / 16$ & 480 & 636 & 28.0 & 81.0 & 116 & 35.5 \\
\hline Warren Elementary ${ }^{1}$ & $12 / 2 / 16$ & $*$ & $*$ & - & - & - & - \\
\hline Williams Creek & $12 / 3 / 16$ & 55.5 & 65.1 & 15.9 & $*$ & 10.7 & - \\
\hline Soil & & $\begin{array}{c}\text { Sample } \\
\# 1(\text { ng/g) }\end{array}$ & $\begin{array}{c}\text { Sample } \\
\# 2 \text { (ng/g) }\end{array}$ & $\begin{array}{c}\% \\
\text { difference }\end{array}$ & $\begin{array}{c}\text { Sample } \\
\# 1 \text { (ng/g) }\end{array}$ & $\begin{array}{c}\text { Sample } \\
\# 2 \text { (ng/g) }\end{array}$ & $\begin{array}{c}\% \\
\text { difference } \\
\end{array}$ \\
\hline Veto Road & $12 / 2 / 16$ & $*$ & $*$ & - & - & - & - \\
\hline Veto Lake & $3 / 25 / 18$ & 9.3 & 10.3 & 10.2 & 1.0 & 1.2 & 18.2 \\
\hline
\end{tabular}

${ }^{1}$ Public water, Warren Community Water.

* Detected but below the LOQ of $10 \mathrm{ng} / \mathrm{L}$ for water or $1 \mathrm{ng} / \mathrm{g}$ for soil.

- Not detected. 
Table S3. Measured PFAS surface water concentration results from the first sampling trip, June 6-9, 2016.

\begin{tabular}{|c|c|c|c|c|c|c|c|c|c|c|c|}
\hline Description & $\begin{array}{c}\text { PFBA } \\
\text { ng/L }\end{array}$ & $\begin{array}{c}\mathrm{PFPeA} \\
\text { ng/L }\end{array}$ & $\begin{array}{l}\text { PFBS } \\
\text { ng/L }\end{array}$ & $\begin{array}{c}\text { PFHxA } \\
\text { ng/L }\end{array}$ & $\begin{array}{c}\text { PFHpA } \\
\text { ng/L }\end{array}$ & $\begin{array}{c}\text { PFHxS } \\
\text { ng/L }\end{array}$ & $\begin{array}{c}\mathrm{PFOA} \\
\mathrm{ng} / \mathrm{L}\end{array}$ & $\begin{array}{c}\text { PFNA } \\
\text { ng/L }\end{array}$ & $\begin{array}{c}\text { PFOS } \\
\text { ng/L }\end{array}$ & $\begin{array}{c}\text { PFDA } \\
\text { ng/L }\end{array}$ & $\begin{array}{c}\text { HFPO- } \\
\text { DA } \\
\text { ng/L }\end{array}$ \\
\hline
\end{tabular}

\section{Ohio River Samples}

Crab Creek
Lakin
Pomeroy
Brinker Run
outflow
Foam in river
Letart
Lee Creek
outflow
Upstream from
Lee Creek
Tuppers Plains
Lubeck
Sawyer Run
Sawyer Run
(dup.)
Belpre
Vienna Island
Vienna Island
(dup.)
Marietta
Newport

$\begin{array}{cc}* & * \\ * & * \\ * & * \\ * & * \\ * & * \\ * & * \\ * & * \\ * & * \\ * & * \\ * & * \\ * & * \\ * & * \\ * & * \\ * & * \\ * & * \\ * & * \\ 10.9 & *\end{array}$

\section{Tributaries Near Known Landfills}

\begin{tabular}{|c|c|c|c|c|c|c|c|c|c|c|c|}
\hline Brinker Run ${ }^{1}$ & * & $*$ & $*$ & $*$ & * & $*$ & 103 & $*$ & - & $*$ & - \\
\hline Lee Creek ${ }^{2}$ & $*$ & $*$ & $*$ & $*$ & $*$ & $*$ & 50.9 & $*$ & $*$ & $*$ & $*$ \\
\hline $\begin{array}{l}\text { Lee Creek }{ }^{2} \\
\text { (dup.) }\end{array}$ & * & * & $*$ & * & $*$ & $*$ & 65.3 & $*$ & $*$ & $*$ & $*$ \\
\hline Pine Run ${ }^{2}$ & * & $*$ & $*$ & $*$ & $*$ & $*$ & 189 & $*$ & $*$ & $*$ & $*$ \\
\hline Vaughts Run ${ }^{3}$ & 27.1 & 16.7 & $*$ & 19.8 & 33.8 & $*$ & 1250 & $*$ & $*$ & $*$ & 71.2 \\
\hline \multicolumn{12}{|l|}{ Other } \\
\hline $\begin{array}{l}\text { Little Kanawha } \\
\text { River }\end{array}$ & $*$ & $*$ & $*$ & $*$ & $*$ & $*$ & 22.6 & $*$ & $*$ & $*$ & - \\
\hline $\begin{array}{l}\text { E. Fork Little } \\
\text { Hocking }\end{array}$ & 10.4 & 15.5 & $*$ & 14.6 & 24.0 & $*$ & 463 & $*$ & $*$ & $*$ & 59.4 \\
\hline $\begin{array}{l}\text { W. Fork Little } \\
\text { Hocking }\end{array}$ & $*$ & $*$ & $*$ & $*$ & $*$ & $*$ & 209 & $*$ & - & * & $*$ \\
\hline $\begin{array}{l}\text { Muskingum } \\
\text { River }\end{array}$ & $*$ & $*$ & * & $*$ & $*$ & $*$ & * & $*$ & * & $*$ & - \\
\hline
\end{tabular}

${ }^{1}$ Influenced by Letart Landfill. ${ }^{2}$ Influenced by Dry Run Landfill. ${ }^{3}$ Influenced by Local Landfill.

* Detected but below the LOQ of $10 \mathrm{ng} / \mathrm{L}$.

- Not detected. 
Table S4. Measured PFAS surface water concentration results from the second sampling trip, July 12-13, 2016.

\begin{tabular}{|c|c|c|c|c|c|c|c|c|c|c|c|}
\hline Description & $\begin{array}{l}\text { PFBA } \\
\text { ng/L }\end{array}$ & $\begin{array}{l}\text { PFPeA } \\
\text { ng/L }\end{array}$ & $\begin{array}{c}\text { PFBS } \\
\mathrm{ng} / \mathrm{L}\end{array}$ & $\begin{array}{c}\text { PFHxA } \\
\text { ng/L }\end{array}$ & $\begin{array}{l}\text { PFHpA } \\
\text { ng/L }\end{array}$ & $\begin{array}{l}\text { PFHxS } \\
\text { ng/L }\end{array}$ & $\begin{array}{l}\text { PFOA } \\
\text { ng/L }\end{array}$ & $\begin{array}{l}\text { PFNA } \\
\text { ng/L }\end{array}$ & $\begin{array}{c}\text { PFOS } \\
\text { ng/L }\end{array}$ & $\begin{array}{l}\text { PFDA } \\
\mathrm{ng} / \mathrm{L}\end{array}$ & $\begin{array}{c}\text { HFPO- } \\
\text { DA } \\
\text { ng/L }\end{array}$ \\
\hline Whites Run \#1 & 36.0 & - & - & 26.8 & 32.2 & - & 1120 & - & - & - & 19.1 \\
\hline Whites Run \#2 & 57.5 & $*$ & - & 34.0 & 38.0 & - & 1290 & - & - & - & 22.7 \\
\hline Sawyer Run \#1 & 31.8 & 10.1 & - & 13.9 & $*$ & - & 421 & - & 10.4 & - & 39.2 \\
\hline West Fork LH & 41.0 & - & - & 18.6 & 21.0 & - & 838 & - & - & - & 49.1 \\
\hline LH \#1 & 50.5 & $*$ & - & 20.3 & 19.2 & - & 902 & - & * & - & 53.1 \\
\hline Little West Branch & 43.0 & $*$ & - & 22.1 & 22.3 & - & 988 & - & * & - & 36.5 \\
\hline LH \#2 & 86.3 & 16.0 & - & 32.8 & 23.7 & - & 1040 & - & * & - & 60.8 \\
\hline LH \#3 & 74.2 & 12.0 & - & 30.4 & 19.5 & - & 1030 & - & - & - & 71.8 \\
\hline Long Brook & 68.4 & $*$ & - & 39.0 & 33.6 & - & 1430 & - & - & - & 84.3 \\
\hline LH \#4 & 136 & 25.7 & - & 56.3 & 59.6 & - & 1770 & - & * & - & 86.2 \\
\hline LH \#4 (dup.) & 85.6 & 11.8 & - & 39.3 & 36.0 & - & 1220 & - & * & - & 36.8 \\
\hline $\mathrm{LH} \# 5$ & 104 & 16.7 & - & 45.3 & 40.2 & - & 1390 & - & * & - & 41.5 \\
\hline Veto Lake & 95.2 & 14.4 & - & 34.4 & 25.7 & - & 932 & - & $*$ & - & 52.6 \\
\hline LH \#6 & 91.8 & 15.2 & - & 40.1 & 42.4 & - & 1360 & - & - & - & 70.0 \\
\hline Mill Branch \#1 & 134 & 19.9 & - & 71.3 & 65.9 & - & 2040 & - & - & - & 115 \\
\hline Davis Creek & 203 & 43.1 & - & 95.0 & 93.7 & - & 2570 & - & 13.0 & - & 227 \\
\hline Mill Branch \#2 & 151 & 22.0 & - & 71.7 & 72.9 & - & 2030 & - & $*$ & - & 88.5 \\
\hline LH \#7 & 91.0 & $*$ & - & 30.1 & 36.2 & - & 987 & - & * & - & 56.8 \\
\hline $\begin{array}{l}\text { E. Branch Tributary } \\
\# 1\end{array}$ & 90.9 & $*$ & - & 39.1 & 32.2 & - & 1200 & - & - & - & 37.4 \\
\hline $\begin{array}{l}\text { E. Branch Tributary } \\
\# 2\end{array}$ & 108 & $*$ & - & 34.8 & 34.5 & - & 1290 & - & - & - & 44.2 \\
\hline $\begin{array}{l}\text { E. Branch Tributary } \\
\# 3\end{array}$ & 80.9 & - & - & 22.3 & 19.2 & - & 962 & - & - & - & 24.8 \\
\hline Browns Run & 106 & $*$ & - & 26.1 & 18.8 & 78.4 & 781 & - & 71.9 & - & 30.4 \\
\hline Briscoe Run & 118 & 28.7 & - & 35.8 & 30.5 & 79.0 & 566 & - & 58.3 & - & - \\
\hline Pond Run & 90.0 & 65.7 & - & 43.2 & 17.8 & 64.5 & 496 & - & 22.1 & - & - \\
\hline Congress Creek & 144 & 11.0 & - & 26.4 & 24.5 & - & 849 & - & 20.0 & - & - \\
\hline
\end{tabular}

* Detected but below the LOQ of $10 \mathrm{ng} / \mathrm{L}$.

- Not detected. 
Table S5. Measured PFAS surface water concentration results from the third sampling trip, December 1-3, 2016.

\begin{tabular}{|c|c|c|c|c|c|c|c|c|c|c|c|}
\hline Description & $\begin{array}{c}\text { PFBA } \\
\text { ng/L }\end{array}$ & $\begin{array}{l}\text { PFPeA } \\
\text { ng/L }\end{array}$ & $\begin{array}{c}\text { PFBS } \\
\text { ng/L }\end{array}$ & $\begin{array}{l}\text { PFHxA } \\
\text { ng/L }\end{array}$ & $\begin{array}{l}\text { PFHpA } \\
\text { ng/L }\end{array}$ & $\begin{array}{l}\text { PFHxS } \\
\text { ng/L }\end{array}$ & $\begin{array}{l}\text { PFOA } \\
\mathrm{ng} / \mathrm{L}\end{array}$ & $\begin{array}{l}\text { PFNA } \\
\text { ng/L }\end{array}$ & $\begin{array}{c}\text { PFOS } \\
\text { ng/L }\end{array}$ & $\begin{array}{l}\text { PFDA } \\
\text { ng/L }\end{array}$ & $\begin{array}{c}\mathrm{HFPO}- \\
\mathrm{DA} \\
\mathrm{ng} / \mathrm{L}\end{array}$ \\
\hline Little Hocking & 19.9 & 10.6 & $*$ & 17.3 & 35.9 & $*$ & 1110 & $*$ & $*$ & $*$ & 129 \\
\hline Long Brook & $*$ & $*$ & $*$ & 10.8 & $*$ & - & 516 & - & - & - & 24.7 \\
\hline Veto Lake & 15.9 & $*$ & $*$ & 11.3 & 10.4 & - & 412 & $*$ & - & - & 31.0 \\
\hline Veto Lake (dup.) & 10.1 & $*$ & $*$ & $*$ & 11.1 & - & 321 & $*$ & $*$ & - & 37.5 \\
\hline Buffalo Run & $*$ & $*$ & - & $*$ & - & - & 157 & $*$ & $*$ & - & 12.9 \\
\hline S. Branch Wolf Creek \#1 & 11.2 & * & $*$ & $*$ & - & $*$ & 98.5 & $*$ & - & - & 14.4 \\
\hline S. Branch Wolf Creek \#2 & $*$ & * & $*$ & $*$ & * & - & 135 & - & - & - & $*$ \\
\hline S. Branch Wolf Creek \#3 & 17.0 & $*$ & $*$ & 13.8 & $*$ & $*$ & 147 & $*$ & - & - & 42.1 \\
\hline S. Branch Wolf Creek \#4 & $*$ & $*$ & - & $*$ & $*$ & - & 151 & $*$ & - & - & $*$ \\
\hline W. Branch Wolf Creek & $*$ & * & $*$ & $*$ & $*$ & - & 22.4 & $*$ & - & - & $*$ \\
\hline Coal Run & $*$ & * & - & $*$ & - & $*$ & 17.7 & - & - & - & $*$ \\
\hline $\begin{array}{l}\text { W. Branch Little Hocking } \\
\# 1\end{array}$ & $*$ & * & - & $*$ & - & - & 32.5 & $*$ & - & - & * \\
\hline $\begin{array}{l}\text { W. Branch Little Hocking } \\
\# 2\end{array}$ & $*$ & * & - & $*$ & - & - & 57.9 & - & - & - & * \\
\hline Big Run $(\mathrm{OH})$ & $*$ & * & $*$ & $*$ & 11.4 & - & 969 & $*$ & $*$ & - & 16.8 \\
\hline Davis Creek & 10.1 & $*$ & $*$ & 17.0 & 31.7 & 11.3 & 1310 & $*$ & 10.7 & - & 137 \\
\hline Congress Creek & 18.1 & 10.8 & $*$ & 11.9 & 15.1 & - & 234 & $*$ & $*$ & - & 24.3 \\
\hline Mill Branch & 12.6 & $*$ & $*$ & 16.0 & $*$ & - & 480 & $*$ & - & - & 81.0 \\
\hline Mill Branch (dup.) & 12.1 & $*$ & $*$ & 14.4 & 14.4 & - & 636 & $*$ & - & - & 116 \\
\hline East Branch tributary \#1 & $*$ & $*$ & $*$ & $*$ & $*$ & - & 876 & $*$ & - & - & 16.5 \\
\hline East Branch tributary \#2 & $*$ & $*$ & $*$ & $*$ & $*$ & $*$ & 293 & $*$ & - & - & $*$ \\
\hline Hanna Road creek & $*$ & $*$ & $*$ & $*$ & * & $*$ & 201 & $*$ & $*$ & - & 18.0 \\
\hline Browns Run & 10.2 & $*$ & $*$ & $*$ & $*$ & 14.4 & 167 & $*$ & 14.0 & $*$ & 17.4 \\
\hline Halfway Run & $*$ & $*$ & $*$ & $*$ & $*$ & $*$ & 96.0 & 24.2 & $*$ & $*$ & $*$ \\
\hline Indian Run & $*$ & * & $*$ & $*$ & - & $*$ & 56.3 & $*$ & - & - & $*$ \\
\hline Tupper Creek tributary & $*$ & $*$ & $*$ & $*$ & - & $*$ & 31.1 & $*$ & - & - & $*$ \\
\hline Rainbow Creek & $*$ & $*$ & - & $*$ & - & - & 42.1 & $*$ & - & - & $*$ \\
\hline Cat Creek & $*$ & $*$ & - & $*$ & - & - & 35.1 & - & - & - & $*$ \\
\hline Duck Creek tributary \#1 & $*$ & $*$ & - & $*$ & - & - & $*$ & $*$ & - & - & $*$ \\
\hline Duck Creek tributary \#2 & $*$ & $*$ & $*$ & $*$ & $*$ & $*$ & 54.3 & $*$ & $*$ & - & $*$ \\
\hline Sugar Creek & $*$ & - & - & $*$ & - & - & $*$ & $*$ & - & - & - \\
\hline Eightmile Creek & $*$ & $*$ & $*$ & $*$ & - & $*$ & 14.1 & $*$ & $*$ & - & * \\
\hline Big Run (WV) & $*$ & $*$ & $*$ & $*$ & - & $*$ & 126 & $*$ & - & - & * \\
\hline Little Eightmile Creek & $*$ & - & - & $*$ & - & - & $*$ & - & - & - & - \\
\hline Fifteenmile Creek & $*$ & $*$ & - & $*$ & $*$ & - & 14.5 & - & - & $*$ & - \\
\hline Wingett Run & $*$ & - & - & $*$ & - & - & $*$ & - & - & - & - \\
\hline Mill Run & $*$ & $*$ & $*$ & $*$ & - & $*$ & 32.5 & $*$ & $*$ & - & $*$ \\
\hline Williams Creek & $*$ & $*$ & $*$ & $*$ & $*$ & $*$ & 55.5 & $*$ & - & - & $*$ \\
\hline Williams Creek (dup.) & 10.3 & $*$ & $*$ & $*$ & $*$ & $*$ & 65.1 & $*$ & - & - & 10.7 \\
\hline Briscoe Run & 10.8 & 17.5 & 28.0 & 19.6 & 29.4 & 10.1 & 146 & $*$ & 19.5 & - & $*$ \\
\hline Pond Run & $*$ & $*$ & $*$ & $*$ & $*$ & $*$ & 74.4 & $*$ & - & - & 22.5 \\
\hline
\end{tabular}

* Detected but below the LOQ of $10 \mathrm{ng} / \mathrm{L}$.

- Not detected. 
Table S6. Measured PFAS concentrations in drinking water samples from the three sampling trips. Each private well is located more than 27 kilometers from fluoropolymer production facility, in the direction of prevailing winds to the northeast.

\begin{tabular}{|c|c|c|c|c|c|c|c|c|c|c|c|}
\hline Description & $\begin{array}{l}\text { PFBA } \\
\text { ng/L }\end{array}$ & $\begin{array}{l}\text { PFPeA } \\
\text { ng/L }\end{array}$ & $\begin{array}{l}\text { PFBS } \\
\text { ng/L }\end{array}$ & $\begin{array}{c}\text { PFHxA } \\
\text { ng/L }\end{array}$ & $\begin{array}{l}\text { PFHpA } \\
\text { ng/L }\end{array}$ & $\begin{array}{l}\text { PFHxS } \\
\text { ng/L }\end{array}$ & $\begin{array}{l}\text { PFOA } \\
\text { ng/L }\end{array}$ & $\begin{array}{l}\text { PFNA } \\
\text { ng/L }\end{array}$ & $\begin{array}{c}\text { PFOS } \\
\text { ng/L }\end{array}$ & $\begin{array}{l}\text { PFDA } \\
\text { ng/L }\end{array}$ & $\begin{array}{c}\text { HFPO- } \\
\text { DA } \\
\text { ng/L }\end{array}$ \\
\hline Washington Co. Library ${ }^{1}$ & $*$ & $*$ & $*$ & $*$ & $*$ & $*$ & $*$ & $*$ & $*$ & $*$ & - \\
\hline Little Hocking P.O. ${ }^{2}$ & - & - & - & - & - & - & - & - & - & - & - \\
\hline Barlow Library ${ }^{2}$ & $*$ & $*$ & - & $*$ & - & - & * & - & - & - & $*$ \\
\hline Watertown P.O. ${ }^{2}$ & $*$ & $*$ & - & $*$ & - & - & $*$ & $*$ & $*$ & - & - \\
\hline Warren Elementary ${ }^{3}$ & $*$ & $*$ & $*$ & $*$ & - & * & $*$ & $*$ & $*$ & - & - \\
\hline $\begin{array}{l}\text { Warren Elementary } \\
\text { (dup.) }\end{array}$ & $*$ & $*$ & $*$ & $*$ & - & - & * & $*$ & - & - & - \\
\hline Devola private well & $*$ & $*$ & $*$ & $*$ & - & $*$ & 28.0 & $*$ & - & - & - \\
\hline Lowell private well & $*$ & $*$ & $*$ & $*$ & - & $*$ & 23.3 & $*$ & - & - & - \\
\hline
\end{tabular}

${ }^{1}$ City of Marietta. ${ }^{2}$ Little Hocking Water Association. ${ }^{3}$ Warren Community Water.

* Detected but below the LOQ of $10 \mathrm{ng} / \mathrm{L}$.

- Not detected.

Table S7. Measured PFAS soil concentration results from soil sampling trips (ng/g dry weight). Distance is measured from fluoropolymer production facility.

\begin{tabular}{|c|c|c|c|c|c|c|c|c|c|c|}
\hline Year & Description & $\begin{array}{c}\text { Distance } \\
(\mathrm{km})\end{array}$ & $\begin{array}{c}\text { PFBA } \\
\mathrm{ng} / \mathrm{g}\end{array}$ & $\begin{array}{c}\text { PFPeA } \\
\text { ng/g }\end{array}$ & $\begin{array}{c}\text { PFHxA } \\
\text { ng/g }\end{array}$ & $\begin{array}{c}\text { PFHpA } \\
\text { ng/g }\end{array}$ & $\begin{array}{c}\text { PFOA } \\
n g / g\end{array}$ & $\begin{array}{c}\text { PFNA } \\
\text { ng/g }\end{array}$ & $\begin{array}{c}\text { PFDA } \\
\text { ng/g }\end{array}$ & $\begin{array}{c}\text { HFPO- } \\
\text { DA } \\
\text { ng/g }\end{array}$ \\
\hline \multirow[t]{3}{*}{2004} & Little Hocking A & & 3.57 & 1.17 & 8.37 & 41.5 & 57.3 & * & 2.01 & - \\
\hline & Little Hocking B & & 3.29 & $*$ & 18.1 & 104 & 66.2 & $*$ & 3.34 & - \\
\hline & Little Hocking C & & 3.78 & 2.15 & 12.9 & 57.9 & 59.1 & $*$ & 2.65 & - \\
\hline \multirow[t]{8}{*}{2016} & Drag Strip Road & 4.0 & $*$ & 6.29 & 1.65 & 1.32 & 26.9 & 1.63 & 1.69 & 3.20 \\
\hline & Veto Lake & 8.0 & 1.22 & 48.8 & 3.63 & 7.65 & 18.4 & $*$ & 1.45 & 8.14 \\
\hline & Veto Road & 13.0 & - & - & - & - & $*$ & - & $*$ & - \\
\hline & Veto Road (dup.) & 13.0 & $*$ & - & - & $*$ & $*$ & - & $*$ & - \\
\hline & Strouds Run & 15.3 & $*$ & 9.99 & $*$ & $*$ & 5.70 & $*$ & $*$ & $*$ \\
\hline & Lookout Park & 24.0 & 1.01 & - & - & $*$ & 2.52 & $*$ & $*$ & - \\
\hline & Archers Fork \#1 & 35.3 & $*$ & - & $*$ & $*$ & 6.77 & $*$ & $*$ & - \\
\hline & Archers Fork \#2 & 48.1 & $*$ & - & * & $*$ & 4.96 & $*$ & $*$ & - \\
\hline \multirow[t]{8}{*}{2018} & LHWA & 1.3 & 3.52 & 14.3 & 3.06 & 3.10 & 6.68 & 1.92 & 1.32 & 3.09 \\
\hline & Veto Lake & 8.0 & $*$ & 3.66 & $*$ & $*$ & 9.30 & $*$ & $*$ & 1.00 \\
\hline & Veto Lake (dup.) & 8.0 & $*$ & 15.4 & 1.40 & 1.30 & 10.3 & $*$ & 1.31 & 1.20 \\
\hline & Watertown & 24.3 & $*$ & 1.37 & $*$ & $*$ & 2.86 & - & $*$ & - \\
\hline & Beverly & 32.1 & $*$ & 4.11 & $*$ & * & 11.0 & $*$ & * & - \\
\hline & L. Olive Green & & & & & & & & & \\
\hline & Creek & 39.9 & $*$ & 3.18 & $*$ & $*$ & 4.88 & $*$ & $*$ & - \\
\hline & Reinersville & 45.4 & $*$ & 4.42 & $*$ & $*$ & 7.35 & $*$ & $*$ & - \\
\hline
\end{tabular}

* Detected but below the LOQ of $1 \mathrm{ng} / \mathrm{g}$.

- Not detected. 
Table S8. Sample metadata from the first sampling trip, June 6-9, 2016.

\begin{tabular}{|c|c|c|c|c|c|}
\hline No. & Description & Date & Time & Latitude & Longitude \\
\hline 01 & Crab Creek & June 6, 2016 & $8: 25$ AM & 38.76207 & -82.20162 \\
\hline 02 & Lakin & June 6 & 10:04 AM & 38.96797 & -82.09248 \\
\hline 03 & Pomeroy & June 6 & 10:49 AM & 39.02617 & -82.03087 \\
\hline 04 & Brinker Run outflow & June 6 & 12:48 PM & 38.91000 & -81.91994 \\
\hline 05 & Foam in river & June 6 & 1:04 PM & 38.90876 & -81.92217 \\
\hline 06 & Brinker Run & June 6 & $1: 15 \mathrm{PM}$ & 38.90851 & -81.92284 \\
\hline 07 & Letart & June 6 & 2:28 PM & 38.89235 & -81.92825 \\
\hline 08 & Lee Creek outflow & June 6 & 4:48 PM & 39.14891 & -81.74234 \\
\hline 09 & Lee Creek & June 6 & $4: 58 \mathrm{PM}$ & 39.15089 & -81.73980 \\
\hline 10 & Lee Creek (dup.) & - & - & - & - \\
\hline 11 & $\begin{array}{l}\text { Upstream from Lee } \\
\text { Creek }\end{array}$ & June 6 & 5:21 PM & 39.15760 & -81.74568 \\
\hline 12 & Pine Run & June 6 & 7:09 PM & 39.18248 & -81.70824 \\
\hline 13 & Tuppers Plains & June 8,2016 & $8: 47 \mathrm{AM}$ & 39.08101 & -81.79697 \\
\hline 14 & Lubeck & June 8 & 11:31 AM & 39.23388 & -81.68987 \\
\hline 15 & Sawyer Run & June 8 & $12: 45 \mathrm{PM}$ & 39.25474 & -81.69424 \\
\hline 16 & Sawyer Run (dup.) & - & - & - & - \\
\hline 17 & Vaughts Run & June 8 & $1: 27 \mathrm{PM}$ & 39.23783 & -81.66928 \\
\hline 18 & Belpre & June 8 & 2:38 PM & 39.26714 & -81.58015 \\
\hline 19 & Little Kanawha River & June 8 & 3:52 PM & 39.24797 & -81.52357 \\
\hline 20 & E. Fork Little Hocking & June 8 & 4:37 PM & 39.27692 & -81.70723 \\
\hline 21 & W. Fork Little Hocking & June 8 & 4:48 PM & 39.27902 & -81.71330 \\
\hline 22 & Vienna Island & June 9, 2016 & 8:00 AM & 39.35587 & -81.53681 \\
\hline 23 & Vienna Island (dup.) & - & - & - & - \\
\hline 24 & Marietta & June 9 & 9:17 AM & 39.39790 & -81.47302 \\
\hline 25 & Newport & June 9 & $11: 22 \mathrm{AM}$ & 39.39679 & -81.20914 \\
\hline 26 & Washington Co. Library & June 9 & $12: 26 \mathrm{PM}$ & 39.42426 & -81.45776 \\
\hline 27 & Muskingum River & June 9 & $12: 59 \mathrm{PM}$ & 39.43301 & -81.47214 \\
\hline
\end{tabular}


Table S9. Sample metadata from the second sampling trip, July 12-13, 2016.

\begin{tabular}{|c|c|c|c|c|c|}
\hline No. & Description & Date & Time & Latitude & Longitude \\
\hline 01 & Whites Run \#1 & July 12,2016 & 9:17 AM & 39.24466 & -81.71711 \\
\hline 02 & Whites Run \#2 & July 12 & $9: 39 \mathrm{AM}$ & 39.23133 & -81.72577 \\
\hline 03 & Sawyer Run \#1 & July 12 & $10: 22 \mathrm{AM}$ & 39.25447 & -81.69927 \\
\hline 04 & Sawyer Run \#1 (dup.) & - & - & - & - \\
\hline 05 & Sawyer Run \#2 & July 12 & 10:49 AM & 39.26103 & -81.72844 \\
\hline 06 & West Fork LH & July 12 & 12:02 PM & 39.27911 & -81.71337 \\
\hline 07 & LH \#1 & July 12 & $12: 20 \mathrm{PM}$ & 39.27691 & -81.70724 \\
\hline 08 & Little West Branch & July 12 & $12: 46 \mathrm{PM}$ & 39.28698 & -81.71290 \\
\hline 09 & LH \#2 & July 12 & 12:59 PM & 39.28558 & -81.70922 \\
\hline 10 & LH \#3 & July 12 & 1:55 PM & 39.29399 & -81.68947 \\
\hline 11 & Long Brook & July 12 & $2: 23 \mathrm{PM}$ & 39.29475 & -81.68580 \\
\hline 12 & LH \#4 & July 12 & $2: 50 \mathrm{PM}$ & 39.29283 & -81.68298 \\
\hline 13 & LH \#4 (dup.) & - & - & - & - \\
\hline 14 & $\mathrm{LH} \# 5$ & July 12 & 4:30 PM & 39.29639 & -81.66705 \\
\hline 15 & Veto Lake & July 12 & 5:59 PM & 39.34101 & -81.65482 \\
\hline 16 & Little Hocking P. O. & July 13,2016 & 4:17 PM & 39.26155 & -81.69953 \\
\hline 17 & LH \#6 & July 13 & 4:42 PM & 39.30514 & -81.65685 \\
\hline 18 & Mill Branch \#1 & July 13 & $4: 52$ PM & 39.29965 & -81.64342 \\
\hline 19 & Davis Creek & July 13 & 5:02 PM & 39.29064 & -81.63391 \\
\hline 20 & Davis Creek (dup.) & - & - & - & - \\
\hline 21 & Mill Branch \#2 & July 13 & 5:22 PM & 39.30770 & -81.61228 \\
\hline 22 & LH \#7 & July 13 & 5:43 PM & 39.33668 & -81.63584 \\
\hline 23 & E. Branch Tributary \#1 & July 13 & 5:54 PM & 39.33463 & -81.63522 \\
\hline 24 & E. Branch Tributary \#2 & July 13 & 6:14 PM & 39.33783 & -81.60300 \\
\hline 25 & E. Branch Tributary \#3 & July 13 & $6: 25$ PM & 39.35571 & -81.57511 \\
\hline 26 & Browns Run & July 13 & 6:46 PM & 39.39378 & -81.58715 \\
\hline 27 & Briscoe Run & July 13 & $7: 23 \mathrm{PM}$ & 39.34695 & -81.53130 \\
\hline 28 & Pond Run & July 13 & 7:36 PM & 39.31056 & -81.54974 \\
\hline 29 & Congress Creek & July 13 & 7:57 PM & 39.28463 & -81.57816 \\
\hline
\end{tabular}


Table S10. Sample metadata from the third sampling trip, December 1-3, 2016.

\begin{tabular}{|c|c|c|c|c|c|}
\hline No. & Description & Date & Time & Latitude & Longitude \\
\hline 01 & Little Hocking & December 1, 2016 & $8: 58 \mathrm{AM}$ & 39.29453 & -81.68806 \\
\hline 02 & Long Brook & December 1 & $9: 16 \mathrm{AM}$ & 39.31497 & -81.67335 \\
\hline 03 & Veto Lake & December 1 & 9:41 AM & 39.34287 & -81.65352 \\
\hline 04 & Veto Lake (dup.) & - & - & - & - \\
\hline 05 & Buffalo Run & December 1 & $10: 26 \mathrm{AM}$ & 39.36601 & -81.65132 \\
\hline 06 & Barlow Library & December 1 & $10: 59 \mathrm{AM}$ & 39.39548 & -81.66668 \\
\hline 07 & S. Branch Wolf Creek \#1 & December 1 & 11:10 AM & 39.40085 & -81.66523 \\
\hline 08 & S. Branch Wolf Creek \#2 & December 1 & 11:57 AM & 39.46452 & -81.63381 \\
\hline 09 & Watertown P.O. & December 1 & $12: 10 \mathrm{PM}$ & 39.46672 & -81.63335 \\
\hline 10 & S. Branch Wolf Creek \#3 & December 1 & 12:27 PM & 39.52470 & -81.65861 \\
\hline 11 & S. Branch Wolf Creek \#4 & December 1 & 1:04 PM & 39.42754 & -81.70965 \\
\hline 12 & W. Branch Wolf Creek & December 1 & 1:34 PM & 39.47943 & -81.73095 \\
\hline 13 & Coal Run & December 1 & 2:09 PM & 39.45974 & -81.82304 \\
\hline 14 & W. Branch Little Hocking \#1 & December 1 & 2:59 PM & 39.38334 & -81.78522 \\
\hline 15 & W. Branch Little Hocking \#2 & December 1 & $3: 32 \mathrm{PM}$ & 39.35959 & -81.73612 \\
\hline 16 & Big Run $(\mathrm{OH})$ & December 1 & 4:06 PM & 39.30150 & -81.72783 \\
\hline 17 & Davis Creek & December 1 & 4:26 PM & 39.29057 & -81.63395 \\
\hline 18 & Congress Creek & December 1 & 4:59 PM & 39.28467 & -81.57794 \\
\hline 19 & Mill Branch & December 2, 2016 & 7:54 AM & 39.30763 & -81.61226 \\
\hline 20 & Mill Branch (dup.) & - & - & - & - \\
\hline 21 & East Branch tributary \#1 & December 2 & $8: 19$ AM & 39.33774 & -81.60314 \\
\hline 22 & East Branch tributary \#2 & December 2 & $8: 45 \mathrm{AM}$ & 39.35566 & -81.57512 \\
\hline 23 & Hanna Road creek & December 2 & $9: 21 \mathrm{AM}$ & 39.38255 & -81.50297 \\
\hline 24 & Warren Elementary & December 2 & $10: 02 \mathrm{AM}$ & 39.39388 & -81.55533 \\
\hline 25 & Warren Elementary (dup.) & - & - & - & - \\
\hline 26 & Browns Run & December 2 & 10:21 AM & 39.39375 & -81.58713 \\
\hline 27 & Halfway Run & December 2 & $10: 55 \mathrm{AM}$ & 39.42693 & -81.57279 \\
\hline 28 & Indian Run & December 2 & $12: 43 \mathrm{PM}$ & 39.43417 & -81.47981 \\
\hline 29 & Devola private well & December 2 & 1:05 PM & 39.46812 & -81.46629 \\
\hline 30 & Tupper Creek tributary & December 2 & 1:15 PM & 39.47305 & -81.46418 \\
\hline 31 & Lowell private well & December 2 & $1: 35 \mathrm{PM}$ & 39.52667 & -81.50190 \\
\hline 32 & Rainbow Creek & December 2 & 2:00 PM & 39.49830 & -81.52533 \\
\hline 33 & Cat Creek & December 2 & 2:24 PM & 39.54458 & -81.51777 \\
\hline 34 & Duck Creek tributary \#1 & December 2 & 3:06 PM & 39.52016 & -81.41509 \\
\hline 35 & Duck Creek tributary \#2 & December 2 & 3:38 PM & 39.42813 & -81.41411 \\
\hline 36 & Sugar Creek & December 2 & 4:10 PM & 39.46746 & -81.39415 \\
\hline 37 & Eightmile Creek & December 2 & 4:36 PM & 39.41497 & -81.34840 \\
\hline 38 & Big Run (WV) & December 3, 2016 & $8: 14$ AM & 39.33147 & -81.47401 \\
\hline 39 & Little Eightmile Creek & December 3 & $8: 49$ AM & 39.44720 & -81.34073 \\
\hline 40 & Fifteenmile Creek & December 3 & 9:33 AM & 39.49367 & -81.29796 \\
\hline 41 & Wingett Run & December 3 & 10:03 AM & 39.54626 & -81.24157 \\
\hline 42 & Mill Run & December 3 & $10: 48 \mathrm{AM}$ & 39.38375 & -81.37831 \\
\hline 43 & Williams Creek & December 3 & $11: 12 \mathrm{AM}$ & 39.39338 & -81.44690 \\
\hline 44 & Williams Creek (dup.) & - & - & - & - \\
\hline 45 & Briscoe Run & December 3 & 11:37 AM & 39.34701 & -81.53130 \\
\hline 46 & Pond Run & December 3 & 11:51 AM & 39.31066 & -81.54963 \\
\hline
\end{tabular}


Table S11. Sample metadata for soil samples.

\begin{tabular}{|c|c|c|c|c|}
\hline Description & Date & Time & Latitude & Longitude \\
\hline $\begin{array}{l}\text { Little Hocking A } \\
\text { Little Hocking B } \\
\text { Little Hocking C }\end{array}$ & $\begin{array}{l}2004 \\
2004 \\
2004 \\
\end{array}$ & & & \\
\hline $\begin{array}{l}\text { Veto Lake } \\
\text { Strouds Run } \\
\text { Drag Strip Road } \\
\text { Veto Road } \\
\text { Veto Road (dup.) } \\
\text { Lookout Park } \\
\text { Archers Fork \#1 } \\
\text { Archers Fork \#2 } \\
\end{array}$ & $\begin{array}{c}\text { December 1, } 2016 \\
\text { December 1, } 2016 \\
\text { December 1, } 2016 \\
\text { December 2, } 2016 \\
- \\
\text { December 2, } 2016 \\
\text { December 3, } 2016 \\
\text { December 3, } 2016 \\
\end{array}$ & $\begin{array}{c}\text { 10:08 AM } \\
\text { 2:43 PM } \\
\text { 4:42 PM } \\
9: 07 \text { AM } \\
- \\
11: 31 \text { AM } \\
9: 10 \text { AM } \\
\text { 10:06 AM } \\
\end{array}$ & $\begin{array}{c}39.34103 \\
39.37151 \\
39.29040 \\
39.34776 \\
- \\
39.41929 \\
39.46487 \\
39.54603 \\
\end{array}$ & $\begin{array}{c}-81.65669 \\
-81.79150 \\
-81.63393 \\
-81.55866 \\
- \\
-81.47079 \\
-81.34868 \\
-81.24160 \\
\end{array}$ \\
\hline $\begin{array}{l}\text { Reinersville } \\
\text { L. Olive Green Creek } \\
\text { Beverly } \\
\text { Watertown } \\
\text { Veto Lake } \\
\text { Veto Lake (dup.) } \\
\text { LHWA }\end{array}$ & $\begin{array}{c}\text { March 25, } 2018 \\
\text { March 25, } 2018 \\
\text { March 25, } 2018 \\
\text { March 25, } 2018 \\
\text { March 25, } 2018 \\
- \\
\text { March 25, } 2018 \\
\end{array}$ & $\begin{array}{c}2: 10 \text { PM } \\
2: 37 \text { PM } \\
2: 59 \text { PM } \\
5: 51 \text { PM } \\
6: 30 \text { PM } \\
- \\
6: 55 \text { PM } \\
\end{array}$ & $\begin{array}{c}39.67770 \\
39.62830 \\
39.55756 \\
39.48623 \\
39.34098 \\
- \\
39.28067 \\
\end{array}$ & $\begin{array}{c}-81.65041 \\
-81.64319 \\
-81.64460 \\
-81.63364 \\
-81.65685 \\
- \\
-81.66475 \\
\end{array}$ \\
\hline
\end{tabular}

